\title{
A STUDY OF THE HEMISPHERIC ASYMMETRY OF SUNSPOT AREA DURING SOLAR CYCLES 23 AND 24
}

\author{
Partha Chowdhury ${ }^{1}$, D. P. Choudhary ${ }^{2}$, and Sanjay Gosain ${ }^{3}$ \\ ${ }^{1}$ University College of Science \& Technology, University of Calcutta, Kolkata 700 009, India; partha240@yahoo.co.in; parthares@ gmail.com \\ ${ }^{2}$ Department of Physics and Astronomy, California State University Northridge, Mail Stop 8268, \\ 18111 Nordhoff Street, Northridge, CA 91330, USA; debiprasad.choudhary@ csun.edu \\ ${ }^{3}$ National Solar Observatory, 950 North Cherry Avenue, Tucson, AZ 85719, USA; sgosain@nso.edu \\ Received 2012 November 26; accepted 2013 March 12; published 2013 April 26
}

\begin{abstract}
Solar activity indices vary over the Sun's disk, and various activity parameters are not considered to be symmetric between the northern and southern hemispheres of the Sun. The north-south asymmetry of different solar indices provides an important clue to understanding subphotospheric dynamics and solar dynamo action, especially with regard to nonlinear dynamo models. In the present work, we study the statistical significance of the north-south asymmetry of sunspot areas for the complete solar cycle 23 (1996-2008) and rising branch of cycle 24 (first 45 months). The preferred hemisphere in each year of cycles 23 and 24 has been identified by calculating the probability of hemispheric distribution of sunspot areas. The statistically significant intermediate-term periodicities of the north-south asymmetry of sunspot area data have also been investigated using Lomb-Scargle and wavelet techniques. A number of short- and mid-term periods including the best-known Rieger one (150-160 days) are detected in cycle 23 and near Rieger-type periods during cycle 24, and most of them are found to be time variable. We present our results and discuss their possible explanations with the help of theoretical models and observations.
\end{abstract}

Key words: Sun: activity - Sun: dynamo - Sun: helioseismology - sunspots

Online-only material: color figures

\section{INTRODUCTION}

The north-south asymmetry is one of the chief characteristics of solar activity and an intensively studied phenomenon. This asymmetry is a basic feature of astrophysical dynamos (Cowling 1934). According to Cowling's theorem, any stationary magnetic field with perfect axial symmetry cannot be maintained by dynamo action, but additional helical non-axisymmetric flow components can contribute to the maintenance of the field which must be variable and asymmetric (Sokoloff \& Nesme-Ribes 1994; Moss et al. 2008). The existence of the $\mathrm{N}-\mathrm{S}$ asymmetry has been investigated in terms of several solar activity indicators such as sunspot numbers and area, group sunspot numbers, solar flares and flare index data, differential rotation, prominences/ filaments, coronal mass ejections, photospheric magnetic flux, coronal Fe lines, etc. (Carbonell et al. 1993; Oliver \& Ballester 1994; Atac \& Özgüc 1996; Gigolashvili et al. 2005; Duchlev 2001; Li et al. 2002, 2009, 2010; Knaack et al. 2004; Gao et al. 2009; Temmer et al. 2002, 2006; Sýkora \& Rybák 2010; Badalyan \& Obridko 2011, and references therein). These studies indicate that asymmetry is a real and systematic phenomenon and is not due to random fluctuations. Observations indicate that the two hemispheres of the Sun show certain asymmetries in the solar-cycle activity indices. For example, during the past cycle 23 , the cycle peak in the north occurred approximately $1.5 \mathrm{yr}$ before the peak in the southern hemisphere. Durrant \& Wilson (2002) reported that the northern hemispheric polar field reversed approximately five months before the southern hemispheric polar field reversal in cycle 23 . Similarly, there are asymmetries in the onset timings, the durations, the amplitudes, and the rise and fall patterns of the cycles in the northern and southern hemispheres. Such features are often lost when the two hemispheres are added together. Therefore, it is very important to analyze the behavior of solar activity separately for the two hemispheres as the indices representing solar activity for the entire disk are the superposition of northern and southern hemi- spheric activities, which may smear out some distinct features like the Gnevyshev gap (Gnevyshev 1963).

Solar cycle 23 (1996 May-2008 December) is the longest $(\sim 12.6 \mathrm{yr})$ in cycle length and its fall time $(\sim 8.8 \mathrm{yr})$ is the longest time for observations of the Sun since the start of modern observations of solar activity cycles. Apart from this, cycle 23 has shown several other peculiarities such as a second maximum during the descending phase which is unusual for odd-numbered cycles; a slower rise to maximum epoch than other oddnumbered cycles; abnormally low activity such that $71 \%-73 \%$ of the days during minima and the rising branch of cycle 24 were entirely spotless. Solar polar field strength measured by the Wilcox Solar Observatory for cycle 23 is reported to be $\sim 50 \%$ lower than for the previous three solar cycles. Kirk et al. (2009) reported a decrease of polar coronal hole $(\mathrm{PCH})$ area by $\sim 15 \%$ during the descending phase of cycle 23 compared to that at the onset of this cycle. Along with using related plasma measurements from Ulysses spacecraft, McComas et al. (2008) have indicated that the solar wind emanating from the large PCHs is slightly slower $(\sim 3 \%)$, significantly less dense $(\sim 17 \%)$, and cooler $(\sim 14 \%)$, with less mass and momentum flux $(\sim 20 \%)$ than previous solar cycle minimum values which has broad effect on the structure and size of the heliosphere. Solar cycle 24 started much later than average, with a weak polar field at the beginning of the cycle, progressing sluggishly.

The structure and evolution of the solar magnetic field is believed to be produced by a dynamo operating inside the Sun's convection zone (Charbonneau 2005) and various activity indices exhibit periodic variations on different timescales ranging from minutes to centuries. For long-term periodicity, the Sun exhibits an $\sim 11$ yr sunspot cycle (Schwabe cycle) and for shortterm variations, the 27 day periodicity is the most prominent. The former is related to the polarity reversal of the solar magnetic field and the latter reflects the modulation imposed on the solar flux at Earth by solar rotation. The regime between these extremes of timescales (between 27 days and $11 \mathrm{yr}$ ) is 
called the "mid range." Apart from these two extremes, several other periodicities such as 150-160 days (Rieger period), 130-180 days (Rieger-type periodicities), $\sim 1.3 \mathrm{yr}$, and quasibiennial oscillations were detected in different solar indices. North-south asymmetry data of various solar activity indices also exhibit different types of periods (Vizoso \& Ballester 1990; Carbonell et al. 1993; Oliver \& Ballester 1994; Knaack et al. 2004; Donner \& Thiel 2007; Badalyan \& Obridko 2011, etc.). In this work, we have investigated the asymmetric behavior of the sunspot area during cycle 23 (1996-2008) and the rising branch of cycle 24 (2009 January-2012 September) and computed its statistical significance. Further, our time-series analysis of the asymmetry data shows the intermediate-term periodicities including Reiger-type periodicities and their time variations.

\section{DATA AND ANALYSIS}

The daily and monthly data of sunspot areas of the full solar disk, and the northern and southern hemispheres of the Sun have been taken from the NASA's Marshall Space Flight Centre (http://solarscience.msfc.nasa.gov/greenwch.shtml). To perform the statistical analysis, the asymmetry $(A)$ has been computed as follows:

$$
A=(N-S) /(N+S)
$$

where $N$ and $S$ respectively are values of sunspot areas in the northern and southern hemispheres.

We have counted the yearly sum of sunspot areas in both hemispheres, calculated the value of asymmetry in each year of cycles 23 and 24 (Table 1) as well as the actual probabilities of generating a $\mathrm{N}-\mathrm{S}$ distribution using paired Student's $t$-test which is a suitable statistical test for a non-integer and dimensional time series (Carbonell et al. 2007) and shows the dominant hemispheres during the entire solar cycle 23 and the ascending phase of cycle 24 (Table 2).

The Student's $t$-test statistics is expressed as follows (Larson 1982; Carbonell et al. 2007):

$$
\hat{t}=\frac{\bar{D}}{S_{\bar{D}}}=\frac{\left(\Sigma D_{i}\right) / n}{\sqrt{\frac{\Sigma D_{i}^{2}-\left(\Sigma D_{i}\right)^{2} / n}{n(n-1)}}},
$$

where $D_{i}$ is the difference of the paired values (in this case monthly values of sunspot areas in northern and southern hemispheres), $n$ is the length of the time series under investigation, $D$ is the average of the $n$ differences, and $s_{\bar{D}}$ is the respective standard deviation. In order to test the statistical significance of the monthly value of asymmetry, we have calculated the statistic $\hat{t}$ based on $n-1$ degrees of freedom, which is compared to the corresponding $\hat{t}_{n-1, \alpha / 2}$ given for a previously chosen probability, $\alpha$. We chose $\alpha=0.05$, i.e., if the difference between paired values is statistically significant at a $95 \%$ level. Thus for each year, the Student's $t$-test is utilized to determine the significance of the difference between the northern and southern hemispheric sunspot areas. If the probability $<0.025$ in the $t$-distribution, then the significance of the N-S asymmetry is denoted by 1 (highly significant, 95\%), otherwise by a "-.."

We fit a regression line to the monthly values of $A$ during the complete solar cycle 23 as well as the initial phase of cycle 24 under investigation. The results are shown in Figures 2(a)-(d). We find that the slope of the straight line changes in each phase, indicating some kind of systematic change in dominance of the activity from one hemisphere to another. Further we divided cycle 23 in two parts viz. from 1996 to 2002 (comprised of the rising phase including maxima) and 2003-2008 (covering the declining phase as well as the minima) and drew the regression curves. This will give better understanding about the change of asymmetry during the ascending and descending phases of cycle 23 .

We have also investigated the short- and mid-term periodicities in asymmetry data for the complete cycle 23 and rising branch of cycle 24 . However, to calculate the periodic behavior of $N-S$ asymmetry data, we have used the absolute value of asymmetry, $\Delta=N-S$, instead of the normalized definition of asymmetry. This is due to the fact that Ballester et al. (2005) have shown that the periodicities obtained by normalized asymmetry time series are misleading as well as changing the general shape of the power spectrum due to the presence of $(N+S)$ in the denominator. Therefore, we have studied short- and intermediate-term oscillations present in the absolute asymmetry $(\Delta)$ data by using the Lomb-Scargle Periodogram (LSP) method (Lomb 1976; Scargle 1982; Horne \& Baliunas 1986; Bai 2003; Ballester et al 2005; Chowdhury \& Ray 2006), in which the power $P_{N}(\omega)$ is defined so that if the signal is pure noise (with zero mean and standard deviation $\sigma=1)$, then the power $P_{N}(\omega)$ follows an exponential probability distribution. This means that for any frequency $\omega_{0}$ the probability that $P_{N}\left(\omega_{0}\right)$ is of height $z$ or higher is $e^{-z}$. Then to estimate the statistical significance of a peak in the power spectrum, we have used false alarming probability (FAP) and considered the significance levels as high as $>90 \%$ $(2 \sigma),>95 \%(3 \sigma),>99 \%(4 \sigma)$, and $>99.99 \%(5 \sigma)$. Side-byside, we have used a complex Morlet wavelet function (WT) $\Psi$ $(\eta)=\pi^{-1 / 4} e^{i w} 0^{\pi} e^{-\eta 2 / 2}$. This is a plane sine wave with amplitude windowed in time by a Gaussian function. Here, $\omega_{0}$ is a non-dimensional frequency and we choose $\omega_{0}=6$ (Torrence \& Compo 1998) which gives reasonable spectral and temporal resolution. The wavelet transform suffers from edge effects at both ends of the time series. This gives rise to a cone of influence (COI). These edge effects, caused by padding zeros at the beginning and at the end of the data series, lead to a power reduction within the COI. The thin black contours within COI show the periods above $95 \%$ confidence level (Torrence $\&$ Compo 1998). The periods which are common in both methods have been considered in our calculation.

\section{OBSERVATIONAL RESULTS}

Figures 2(a)-(c) and Table 1 show that as solar cycle 23 progresses, the southern hemisphere dominates. The present study indicates that the solar activity with respect to sunspot area was dominant in the southern hemisphere during cycle 23 and this result is consistent with the previous observations of $\mathrm{Li}$ et al. (2002, 2009). On the other hand, the northern hemisphere dominates in the initial phase of cycle 24 (cf. Table 1). Figure 1 shows that from 2012 July, the southern hemisphere started to dominate over the northern one corresponding to a gradual shift in the excess of the total unsigned magnetic flux from north to south. The $N-S$ asymmetry value shifted from positive to negative in 2012, but its value is very small. The regression curve for cycle 24 (Figure 2(d)) shows a slight shift in the negative direction. A similar kind of shift of magnetic flux from the northern part to the southern part of the Sun has been found during 2002-2003 (Table 1) around which was the maxima of cycle 23 . However, to investigate the exact period during which the excess magnetic flux trailed from the northern hemisphere to the southern one, we have plotted the cumulative monthly mean northern and southern sunspot areas (Li et al. 2001) for cycle 23 (Figure 3 ) and the graph confirms that around the middle of 


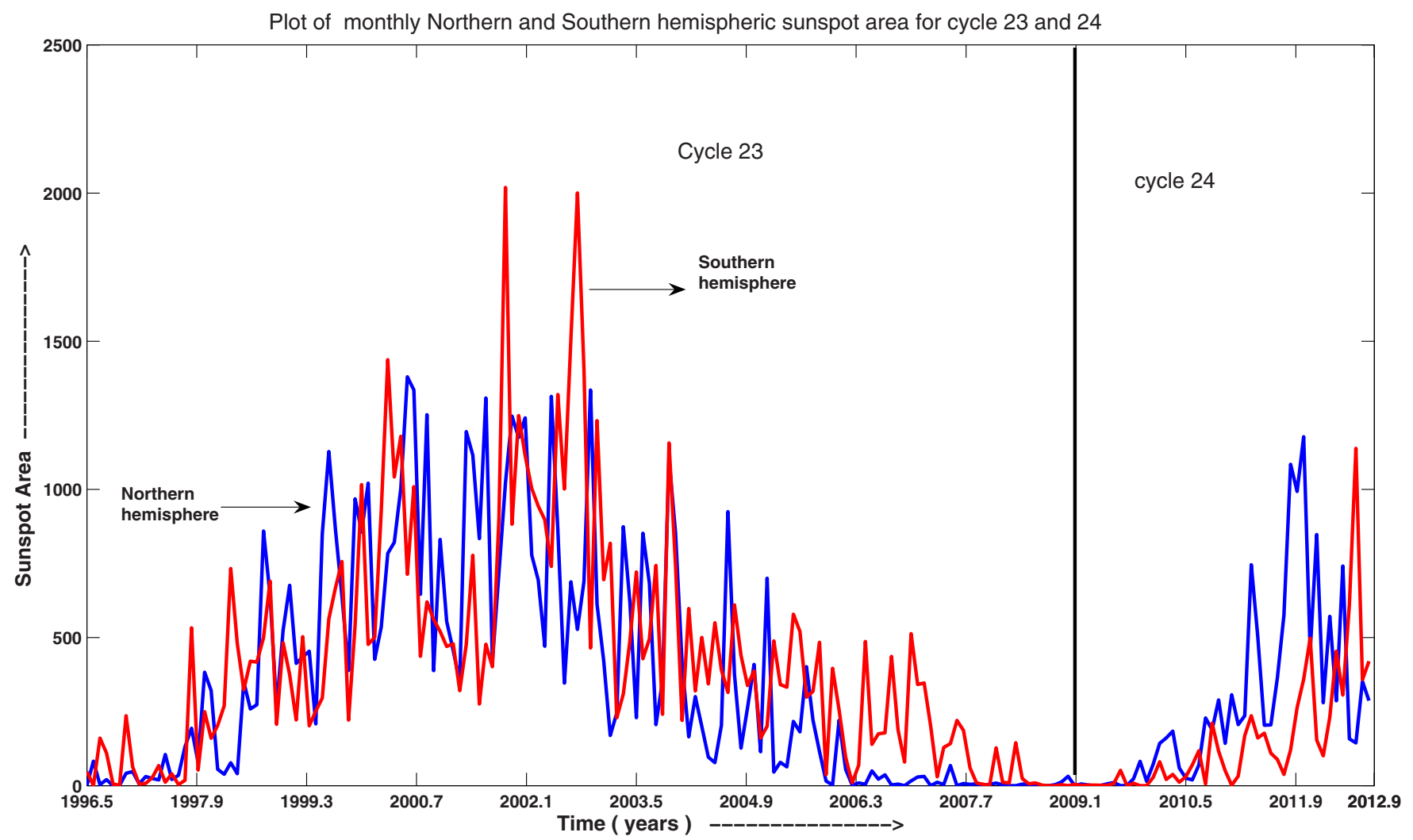

Figure 1. Monthly values of sunspot areas respectively in the northern (blue line) and southern (red line) hemispheres for cycle 23 and the rising phase of cycle 24 .

(A color version of this figure is available in the online journal.)

Table 1

Hemispheric Distribution of Sunspot Area During Cycles 23 and 24

\begin{tabular}{lrrrc}
\hline \hline Year & $N$ & $S$ & $A$ & $\begin{array}{c}\text { Preferred } \\
\text { Hemisphere }\end{array}$ \\
\hline 1996 & 5978 & 19082 & -0.522 & $\mathrm{~S}$ \\
1997 & 41118 & 35476 & 0.073 & $\mathrm{~N}$ \\
1998 & 12422 & 155176 & -0.110 & $\mathrm{~S}$ \\
1999 & 251230 & 173600 & 0.182 & $\mathrm{~N}$ \\
2000 & 302708 & 286216 & 0.028 & $\mathrm{~N}$ \\
2001 & 337744 & 285026 & 0.084 & $\mathrm{~N}$ \\
2002 & 265751 & 402290 & -0.204 & $\mathrm{~S}$ \\
2003 & 201208 & 2017112 & $-1.25 \times 10-3$ & $\mathrm{~S}$ \\
2004 & 99463 & 151263 & -0.206 & $\mathrm{~S}$ \\
2005 & 69636 & 128506 & -0.297 & $\mathrm{~S}$ \\
2006 & 7098 & 82643 & -0.841 & $\mathrm{~S}$ \\
2007 & 4480 & 44156 & -0.815 & $\mathrm{~S}$ \\
2008 & 1834 & 6559 & -0.562 & $\mathrm{~S}$ \\
Total (cycle23) & 1712470 & 1971705 & -0.0703 & $\mathrm{~S}$ \\
2009 (cycle 24) & 6692 & 3108 & 0.365 & $\mathrm{~N}$ \\
2010 (cycle 24) & 55434 & 22984 & 0.413 & $\mathrm{~N}$ \\
2011 & 205362 & 68544 & 0.499 & $\mathrm{~N}$ \\
2012 (January-September) & 112378 & 151752 & -0.012 & $\mathrm{~S}$ \\
\hline
\end{tabular}

2002 was the transition phase. Both the results of cycle 23 and the ascending phase of cycle 24 confirm that asymmetry is in favor of the northern hemisphere at the onset of the cycle, then as the cycle progresses, the southern hemispheric predominance takes over.

Using LSP and WT methods (Figure 4), we find a number of short- and mid-term quasi-periodicities during cycle 23. The main periodicities detected are 24-32 days, 50-60 days, 90-125 days, best-known Rieger periods of $\sim 159$ and $\sim 171$ days, $\sim 1.4 \mathrm{yr}$, and a quasi-biennial period (QBP) of $\sim 2.1 \mathrm{yr}$ in the sunspot area asymmetry data during different phases of cycle 23 . The wavelet power spectrum shows that the Rieger period was present during the interval from the middle of 2001 to the middle of 2003. A large contour extending from $1.2 \mathrm{yr}$ to about $2.2 \mathrm{yr}$ persisted from late-1997 to the middle of 2003.

During cycle 24 (Figure 5a), the significant periods are 20-30 days, $40-70$ days, $\sim 105$ days, near Rieger-type period $\sim 195$ days, and a period $\sim 1$ yr. The wavelet power spectrum (Figure $5 \mathrm{~b}$ ) shows that Rieger-type periods were present from around middle of 2011 to the end of mid-2012. However, after the end of 2011 this period was edge affected. The nearly one-year period exists from the onset of 2011 to the middle of 2012, but is edge affected after mid-2011. To the best of

Table 2

Calculation of Dominant Hemisphere by $t$ Distribution

\begin{tabular}{lccccc}
\hline \hline Solar Cycle & $\begin{array}{c}\text { Sunspot Area in N-Hemisphere } \\
\text { (Monthly Average) }\end{array}$ & $\begin{array}{c}\text { Sunspot area in S-hemisphere } \\
\text { (Monthly Average) }\end{array}$ & Probability & Significance & $\begin{array}{c}\text { Dominant } \\
\text { Hemisphere }\end{array}$ \\
\hline 23 (152 months) & 56155.40 & 64776.00 & $2.08452 \mathrm{e}-002$ & 1 & South \\
24 (first 45 months) & 12444.50 & 6876.70 & $6.54595 \mathrm{e}-003$ & 1 & North \\
\hline
\end{tabular}


(a) Plot of N - S asymmetry of sunspot area for ascending phase including maxima of cycle 23 ( 1.5 .1996 - 31.12 .2002 )
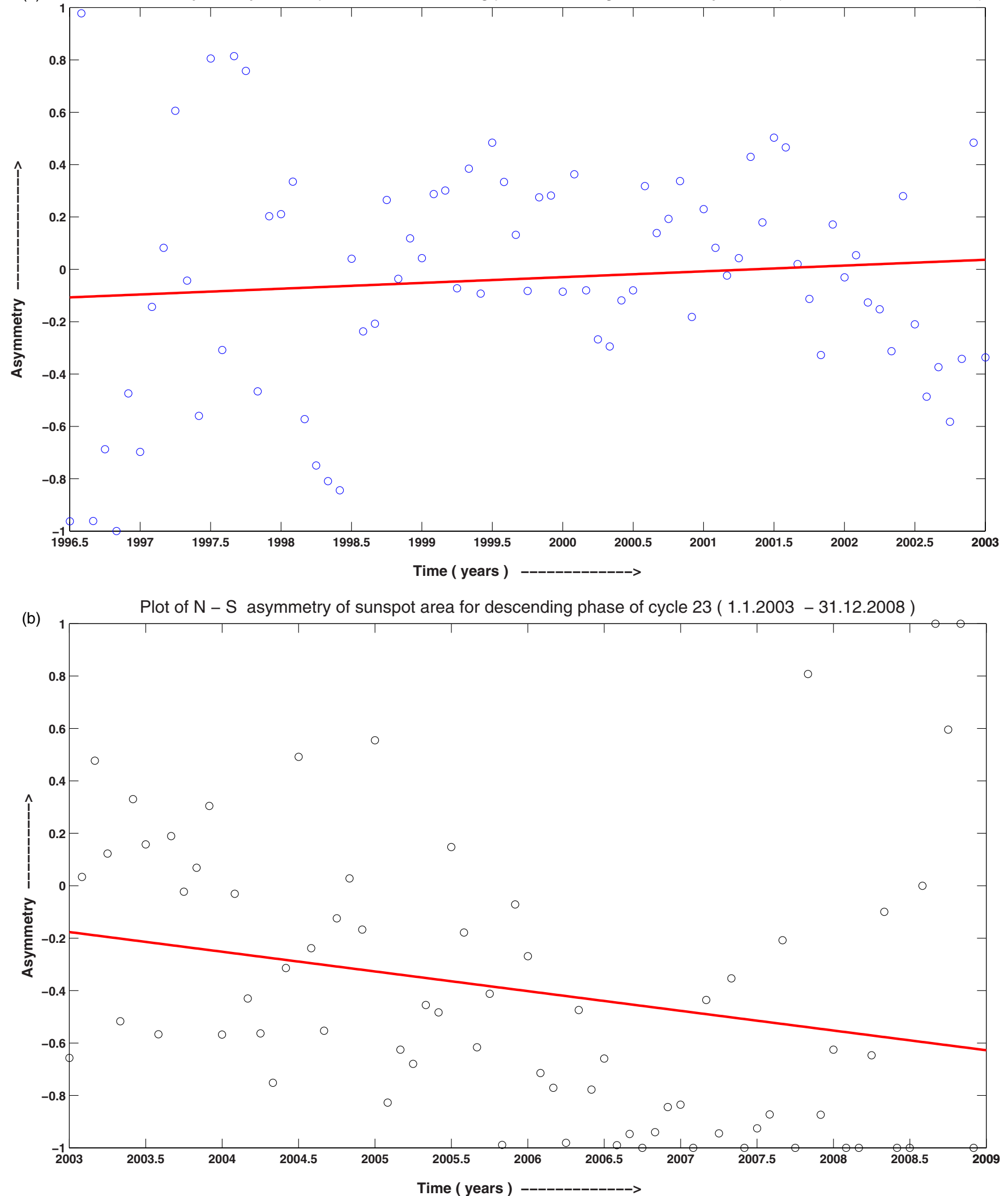

Figure 2. (a) Fit of a regression line to the monthly N-S asymmetry values of sunspot area for the ascending phase of cycle 23. (b) Same as the descending branch of cycle 23. (c) For the full cycle 23 and (d) for the ascending phase of cycle 24 (1.1.2009-30.9.2012).

(A color version of this figure is available in the online journal.) 

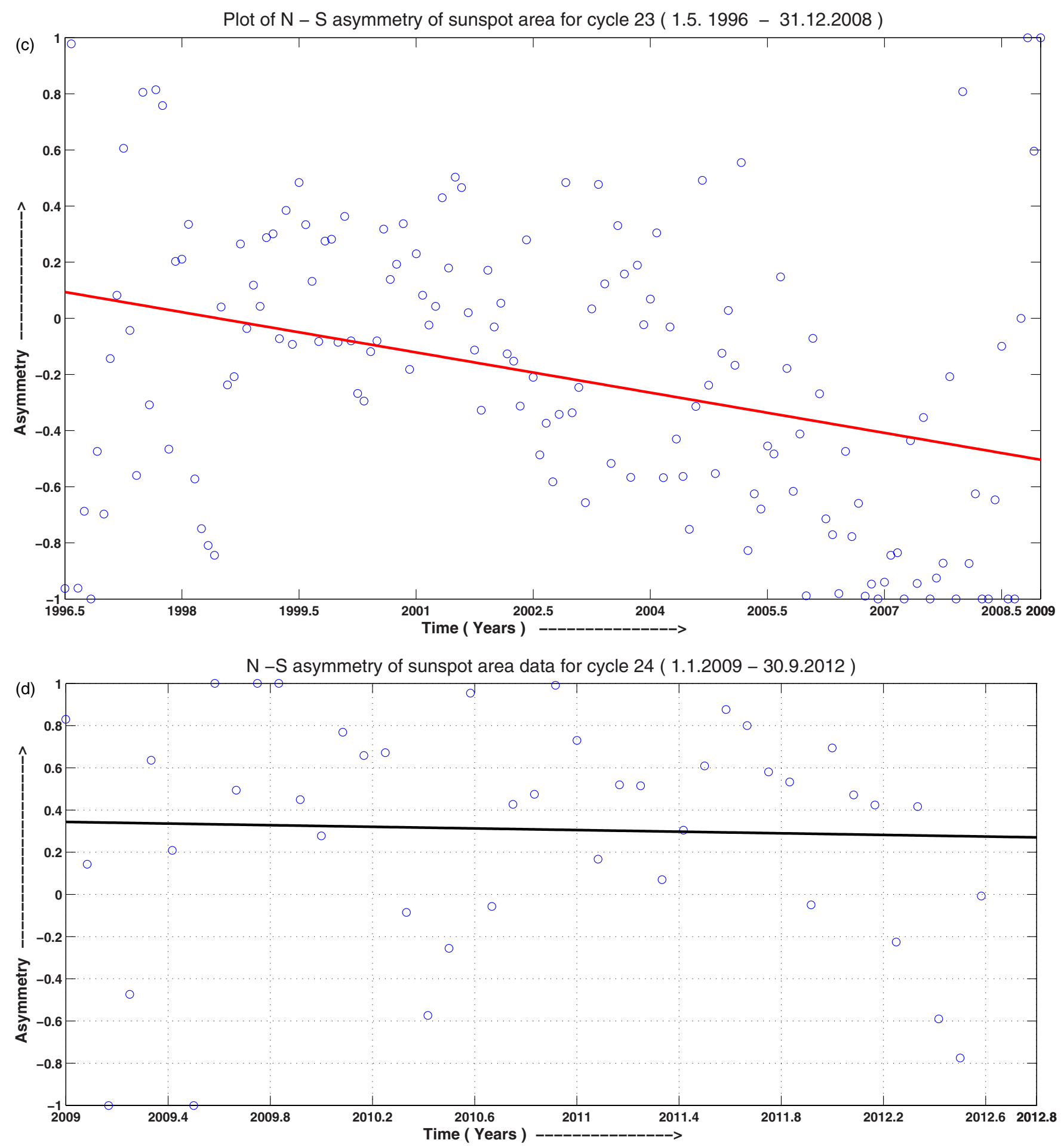

Figure 2. (Continued)

our knowledge, this is the first report detecting Rieger-type periods in $N-S$ asymmetry time series during cycle 24 . These results are consistent with the earlier results in various activity indicators such as group sunspot numbers (Temmer et al. 2006), photospheric magnetic flux (Knaack et al. 2004), coronal Fe lines (Badalyan et al. 2008), etc.

\section{DISCUSSION AND CONCLUSIONS}

Time-series analysis of sunspot area asymmetry data for the complete cycle 23 and initial phase of cycle 24 reveals the presence of a number of quasi-periodicities including the "Rieger"-type one. We have detected a group of periods in the range of 24-32 days which mainly reflects the solar rotational periods. Among the periodic oscillations of solar activities, the Rieger and Rieger-type periodicities (130-180 days) and QBPs were revealed in various indices (Lean \& Brueckner 1989; Pap et al. 1990; Oliver et al. 1992; Bai 2003; Chowdhury \& Ray 2006; Hathaway 2010, etc). However, relatively little work has been done to study the presence of these periods in the asymmetry data. The present investigation shows the presence of a Rieger period and QBPs during cycle 23. Near 


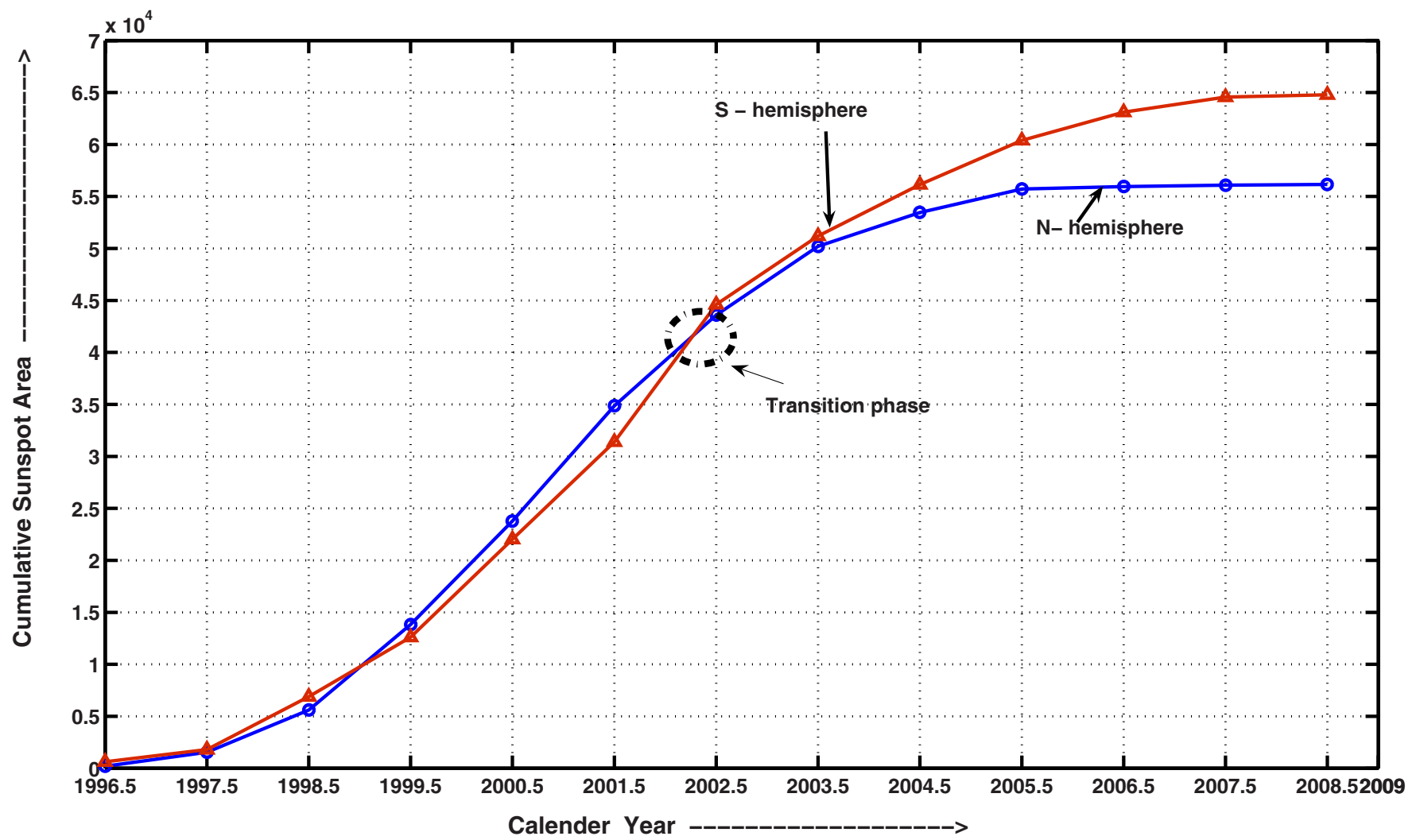

Figure 3. Cumulative sunspot areas at the northern hemisphere (blue lines) and the southern hemisphere (red lines) in cycle 23. The transition phase during which excess magnetic flux changes its sign is denoted by an ellipse.

(A color version of this figure is available in the online journal.)

Rieger-type oscillations has also been detected for the first time in the ascending epoch of cycle 24 . Our analysis shows the occurrence of a period $\sim 1.4$ yr during cycle 23 , which was previously detected in the rotational rate at the base of the solar convection zone (Howe et al. 2000). Chowdhury et al. (2009) have confirmed its presence in the daily data of sunspot areas during cycle 23 , but noticed that this oscillation is concentrated mainly in the southern part of the Sun. Knaack et al. (2004) noticed oscillations in this range in the asymmetry data of photospheric magnetic flux during the interval 1975-2003 and near 2 yr periods were found by Sýkora \& Rybák (2010) in coronal green line oscillations. Simoniello et al. (2012) searched the signatures of QBP over different phases of solar cycle 23 by means of acoustic modes of oscillation ( $p$-mode) and noticed that the strength of the QBP signal is modulated by the $11 \mathrm{yr}$ envelope, but the effect is more significant at low to middle latitudes compared to higher ones. Using GONG data these authors found the signatures of QBP during the minimum phase of solar cycle 23 which is consistent with the present work.

Although physical reasons behind these periodicities are still unknown, they are possibly related to the evolution of complexes of solar activity and their characteristic lifetime. The complexes of activities are produced due to emergence of magnetic flux near or within already created active regions (Ballester et al. 2002) and may persist from 3-6 up to roughly 20-30 consecutive solar rotations (Gaizauskas et al. 2001). Thus, it is possible that at $\sim 1.4 \mathrm{yr}$, QBPs may be related to the lifetime of these complex activities and their independent evolution in one or both solar hemispheres. These periods are also detected in different solar parameters in different time spans and presently are being explained with the help of " $r$ " mode oscillations or Rossby types of waves occurring inside the Sun (Knaack et al. 2005; Chowdhury et al. 2009; Sturrock et al. 1999; Sturrock 2008; Lou 2000, etc). Recently Zaqarashvili et al. (2010a) have studied the behavior of magnetic Rossby waves in the solar tachocline and argued that the joint action of typical differential rotation parameters and toroidal magnetic field with strength $\leqslant 10^{4} \mathrm{G}$ favors the strong growth of the $m=1$ harmonic with a period range of 150-170 days. In another paper, Zaqarashvili et al. (2010b) have considered shallow water MHD approximation and showed that strong magnetic field strength of $\geqslant 10^{5} \mathrm{G}$ triggers the growth of magnetic Rossby wave harmonic with a period of about $2 \mathrm{yr}$. These authors further argued that Rieger and near Rieger-type periods are generated in the upper overshoot layer of the tachocline and QBP is produced in the lower layers under the influence of the strong magnetic field. We consider that detailed knowledge about dynamics of Rossby waves is required to gather full information about the intermediate-term periodicities in solar asymmetry data.

The solar magnetic cycle is believed to be produced by a flux transport dynamo operating in the Sun's highly turbulent convection zone. Fairly sophisticated solar dynamo models that can explain various regular features of the sunspot cycle have been constructed. In the modern flux transport dynamo models (FTD) developed by several scientists (Wang et al. 1991; Choudhuri et al. 1995; Durney 1995; Dikpati \& Charbonneau 1999; Dikpati \& Gilman 2001; Nandy \& Choudhuri 2002; Guerrero \& Muñoz 2004, etc.), three basic processes are combined: (1) the strong toroidal field is produced by the stretching of the poloidal field by differential rotation in the turbulent tachocline, (2) the toroidal field generated in 


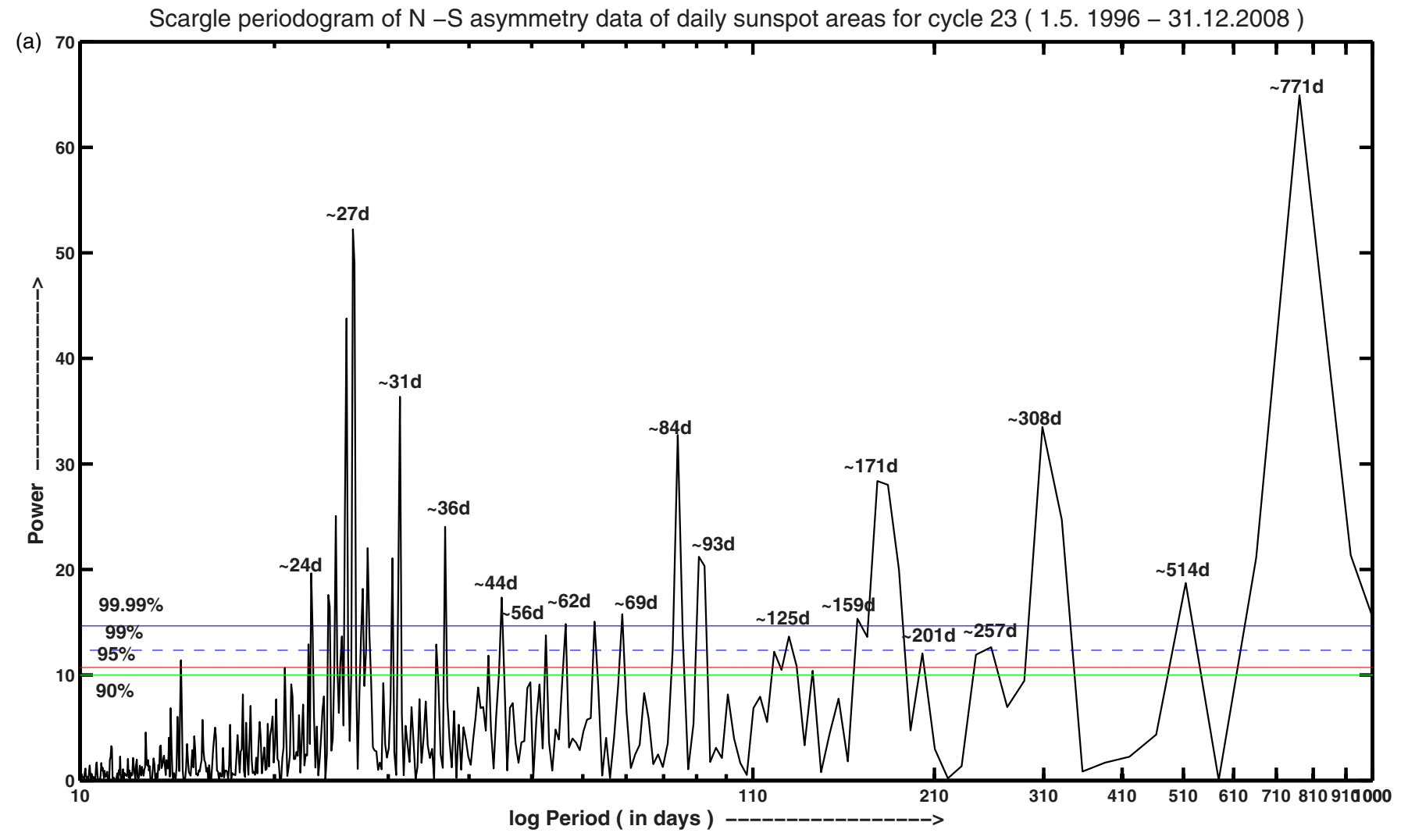

Wavelet Spectrum of $\mathrm{N}-\mathrm{S}$ asymmetry data of sunspot areas for solar cycle $23(1.5 .1996-31.12 .2008)$

(b)

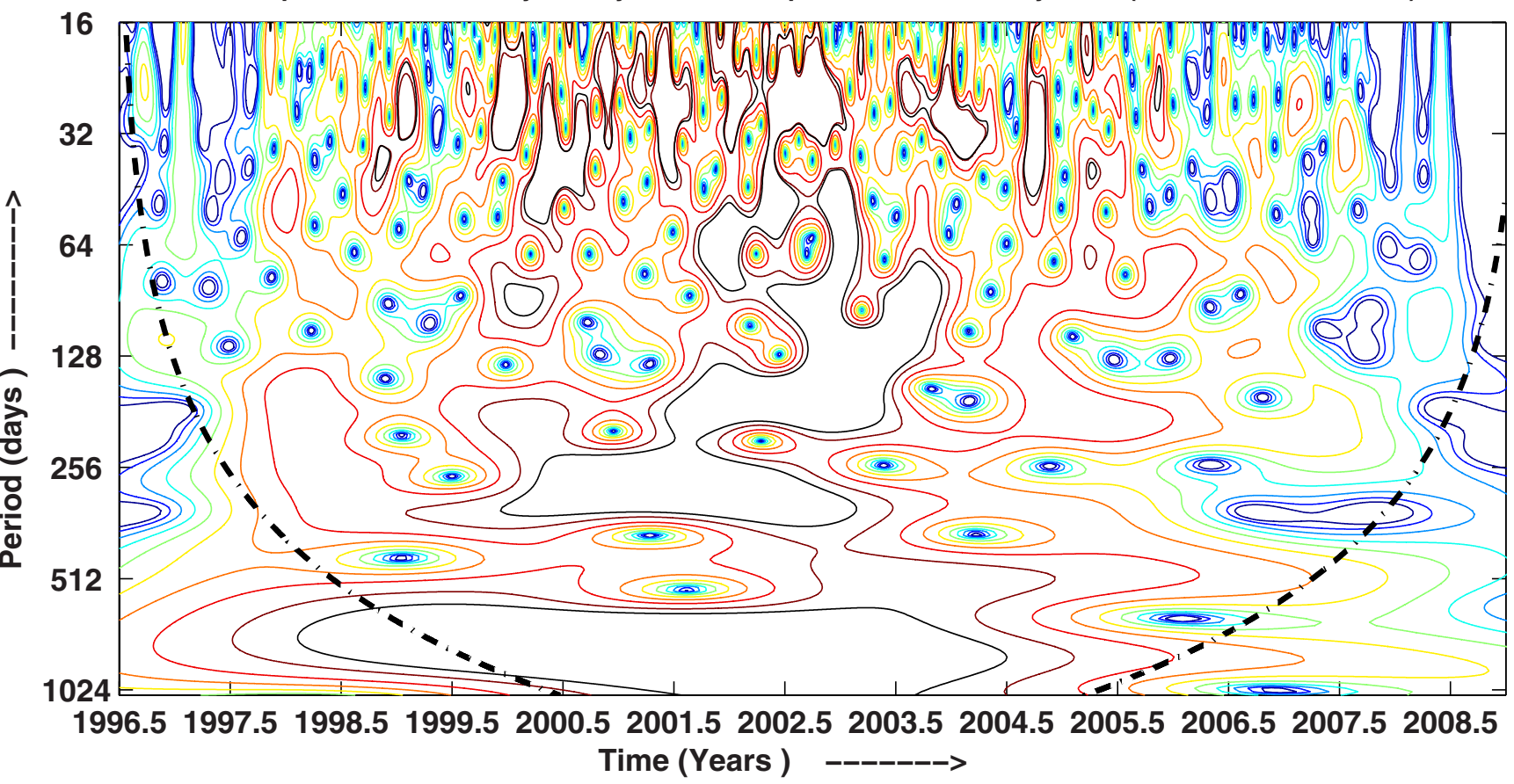

Figure 4. (a) Scargle periodogram of daily N-S asymmetry data during cycle 23 (1.5.96-31.12.2008). (b) Same with Morlet wavelet spectrum. The black contours inside the COI represent the periods above $95 \%$ confidence level.

(A color version of this figure is available in the online journal.)

the convection zone rises upward due to magnetic buoyancy to produce sunspots/active regions, and the decay of tilted bipolar sunspots produces the poloidal field by the Babcock-Leighton mechanism, (3) the meridional circulation advects the poloidal field first to high latitudes and then down to the tachocline at the base of the solar convection zone.
Therefore, the Sun's axisymmetric flows (such as differential rotation and meridional circulation) play key roles in forming the sunspot cycle. The meridional circulation transports the magnetic field from the equator to the poles, builds up the polar field and intensity of the sunspot cycle, and acts as a conveyor belt. On the other hand, differential rotation shears the poloidal 
(a)

(a) Scargle periodogram of $\mathrm{N}-\mathrm{S}$ asymmetry of daily sunspot area for cycle $24(1.1 .2009-30.9 .2012)$

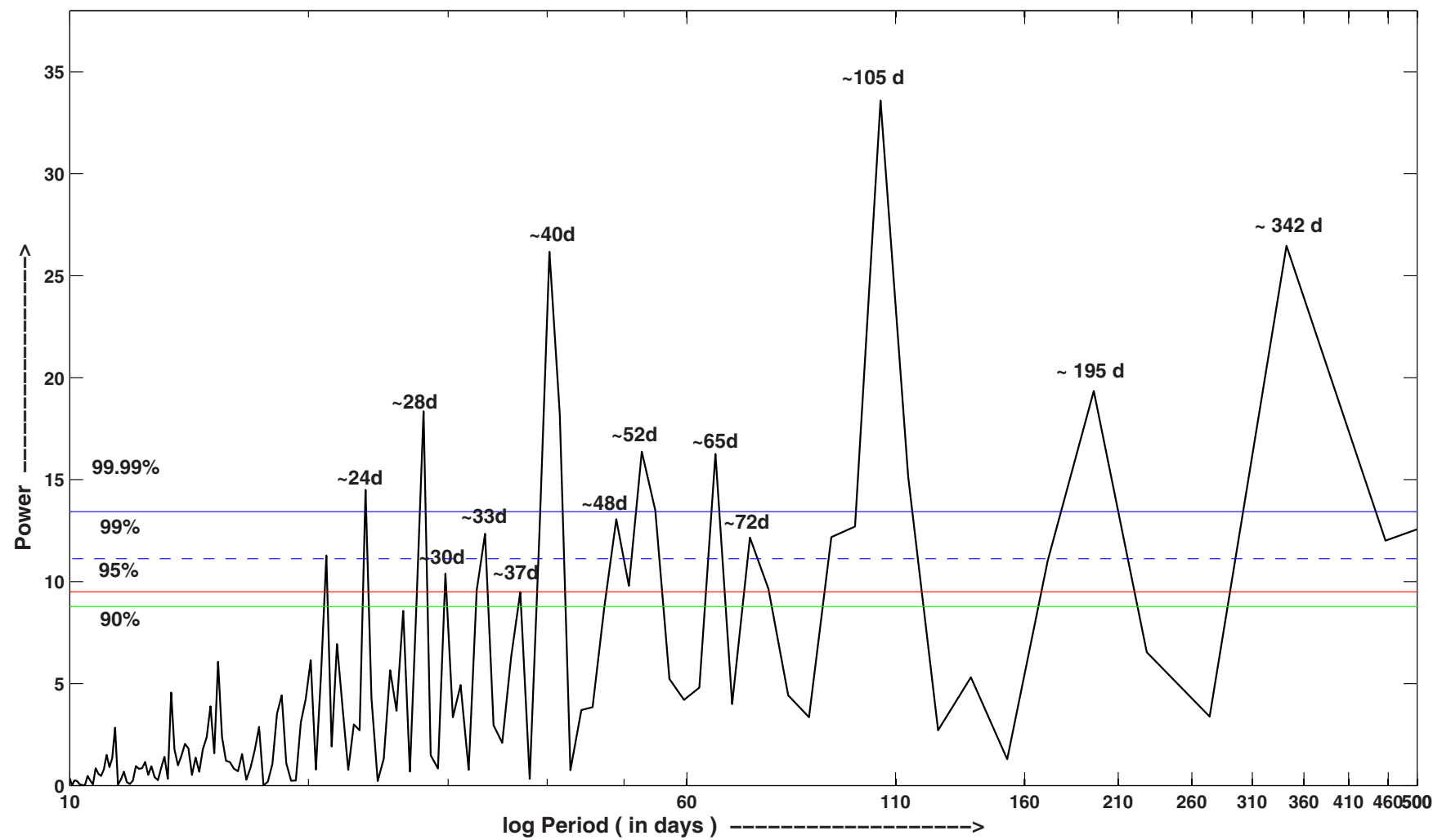

(b) Wavelet Spectrum of N-S asymmetry of daily sunspot area data for cycle $24(1.1 .2009 \times 30.9 .2012)$

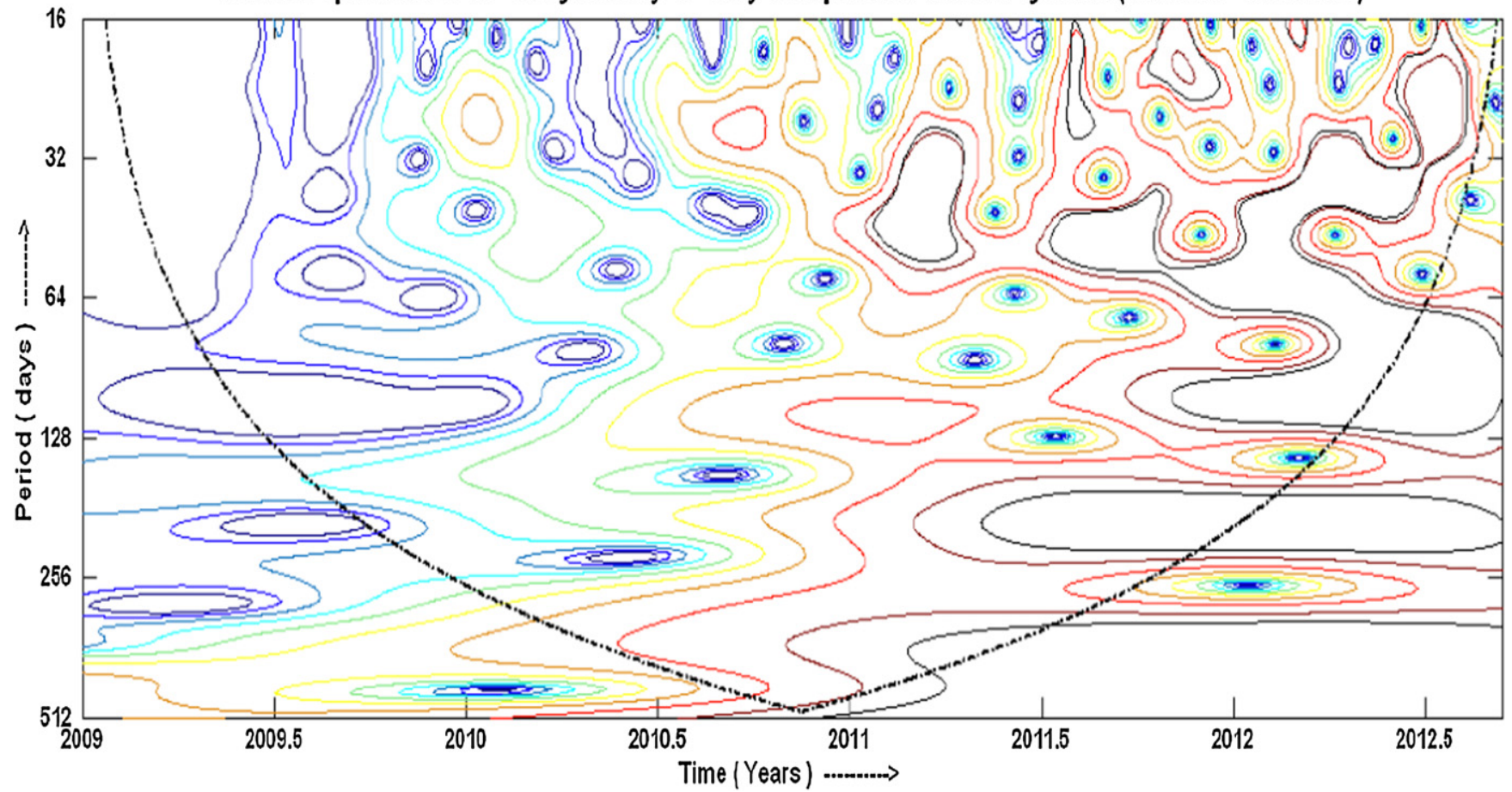

Figure 5. (a) Scargle periodogram of daily N-S asymmetry during cycle 24 (1.1.2009-30.09.2012). (b) Same with Morlet wavelet spectrum. The black contours inside the COI represent the periods above $95 \%$ confidence level.

(A color version of this figure is available in the online journal.)

field to produce strong toroidal fields that erupt through the photosphere in the form of sunspots and active regions. If by any means the $\mathrm{N}-\mathrm{S}$ asymmetry is produced in the polar field during cycle minima, then it would lead to asymmetry in the next solar cycle. Asymmetry in meridional flow would also produce asymmetry in the solar cycle. Dikpati et al. (2004) have identified that north-south asymmetry of the meridional circulation can cause the polar field in one hemisphere to reverse 
before the polar field in the other hemisphere. Choudhuri (1992) considered that the stochastic fluctuations in the dynamo process are a possible reason for the irregularities of the sunspot cycle. Choudhuri et al. (2007) and Jiang et al. (2007) identify the Babcock-Leighton process of the production of the poloidal field as the main source of randomness in the solar dynamo because the convective buffeting on rising flux tubes causes a scatter in the tilt angles, whereas other aspects of the dynamo process are assumed to be deterministic as only $1 \%-2 \%$ of the toroidal field is converted to polar field.

Zaatri et al. (2006), after studying the N-S asymmetry of zonal and meridional components of horizontal solar subsurface flows during 2001-2004 using a ring-diagram analysis of highresolution GONG data, showed that the zonal flows are larger in the southern hemisphere and that this $\mathrm{N}-\mathrm{S}$ asymmetry increases with depth. The average meridional flow has a large amplitude in the southern hemisphere equatorward of the mean latitude of magnetic activity and as a result more sunspots appeared in the southern hemisphere than in the northern one during that interval. Recent measurements of MDI images of the line-of-sight magnetic fields have shown a lot of variation in meridional flow speeds in cycle 23 (Hathaway \& Rightmore 2010). These authors detected a meridional flow speed of $11.5 \mathrm{~ms}^{-1}$ in 1996-1997 (minima of cycle 23) which then dropped to $8.5 \mathrm{~ms}^{-1}$ around maxima (2000-2001) and increased to $13 \mathrm{~ms}^{-1}$ in 2004 and maintained this value thereafter. Janardhan et al. (2010) noticed a large and unusual drop in the absolute value of polar fields during cycle 23 which are well correlated with the variations in meridional flow speeds and indicated that the memory of these weak polar fields is the cause of extended minima in cycle $23 / 24$. Hathaway \& Rightmore (2011) analyzing MDI data have shown $20 \%$ increase of meriodional flow during cycles 23/24 minima compared with the minima of cycle $22 / 23$. It is possible that this faster meridional flow produces weaker polar fields in the solar flux transport models of Schrijver \& Liu (2008) and Wang et al. (2009) and produces a weak coming cycle. Recently, Basu \& Antia (2010) studied solar meridional flows and their variations for cycle 23 and reported that the time dependence of variation is a function of both latitude and depth. We have detected that 2002-2003 is the transition phase of cycle 23, when excess magnetic flux shifted from the northern hemisphere to the southern one and in the similar time interval, Basu \& Antia (2003) have reported a change in zonal flows and that the antisymmetric component of the meridional flow shows a decrease in speed with activity. But they did not found any significant change of the tachocline which is assumed to be the seat of dynamo. Goel \& Choudhuri (2009) assumed that randomness in the Babcock-Leighton process of poloidal field generation to be the main source of irregularity in the sunspot cycle. After studying the asymmetry in the northern and southern polar faculae number they inferred that the $\mathrm{N}-\mathrm{S}$ asymmetry should tend to get reduced as the cycle progresses, and the hemispheric asymmetries should be expected to continuously get washed away until the randomness in the Babcock-Leighton process creates fresh asymmetries toward the end of the cycle. Our findings are in the variance of their inference.

Considering sunspot area data, our results indicate that cycle 24 is $\sim 20 \%$ weaker than cycle 23 until now. This result is consistent with the predictions of Choudhuri et al. (2007) who used polar field strength in cycle minima in the dynamo model with high diffusivity, as well as those of Svalgaard et al. (2005) who considered polar field strength as an indicator of the peak sunspot activity in the next cycle and proposed a weak cycle 24. On the other hand, Dikpati et al. (2006) and later Dikpati \& Gilman (2006) had fed sunspot area and positions in their dynamo code, considered low diffusivity, and predicted that cycle 24 would be $30 \%-50 \%$ stronger than the previous one. However, this matter would be clearer over the next 1-2 yr when cycle 24 will attain its maximum epoch. Observing the present trend, we propose that cycle 24 will be weaker than cycle 23 and its size would be medium.

The origin of hemispheric asymmetry in the solar indices is still not clear. The $\mathrm{N}-\mathrm{S}$ asymmetries might be caused by the phase differences between the magnetic activity in the hemispheres, interference between the dominant dipolar modes and modes with quadrupolar symmetry with respect to the solar equator, and stochastic fluctuations of various parameters such as randomness in the formation and appearance of sunspots at solar surface (Mininni \& Gomez 2002; Ossendrijver 2003). Chatterjee et al. (2004) and Chatterjee \& Choudhuri (2006) studied this problem with FTD and inferred that a dipolar parity is preferred when the magnetic fields in the two hemispheres are coupled sufficiently strongly by a diffusivity on the higher side. They found high diffusivity forced the cycles in the two hemispheres to remain locked with each other even in the presence of asymmetries between the hemispheres. However, on reducing the diffusivity, they found that the solution tended to be quadrupolar. Our result shows a relatively fast variation of asymmetry during different years of cycles 23 and 24 which suggests the relation of asymmetry to fields of quadropole type as suggested by Dikpati \& Gilman (2001). But the question arises as to why the solar magnetic field appears dipolar and why the insertion of additional sources of poloidal field within the solar convection zone make solutions dipolar. It has also been proposed that the dipolar and quadrupolar components of dynamo models could both be oscillating coherently and therefore the observed asymmetry should have a period component to its signal (Brooke et al. 1998). In the future, a better dynamo model which will incorporate the actual value of diffusivity (low or high), exact location (surface, body, or bottom of the convection zone) of the operation of the poloidal field source parameter and a clear explanation of why the magnetic helicity of active regions tends to be negative (positive) in the northern (southern) hemisphere should quantitatively represent the mid-term characteristic timescale, in addition to the $11 \mathrm{yr}$ cycle length.

The present analysis leads us to the following conclusions.

1. The southern hemisphere was dominant during cycle 23 and 2002-2003 (especially mid of 2002) was the transition phase when excess magnetic flux gradually shifted from the northern hemisphere to the southern hemisphere. During maxima of cycle 23 , the asymmetry was very small and is weakly coupled and developed rather independently. During cycle 24 , initially the northern hemisphere was dominating and from 2012 June to July the asymmetry changed its sign. However, to know which hemisphere will dominate ultimately we need to wait until the end of this cycle.

2. After completing the first 45 months, with regard to sunspot area, the present cycle is $\sim 20 \%$ weaker than the preceding cycle.

3. Time series analysis of asymmetry data shows the presence of a Rieger period as well as $\sim 1.4 \mathrm{yr}$ and QBP along with other periods during cycle 23 . During cycle 24 , we have detected near Rieger-type period as well as a period around 1 yr. In both cycles a group of periods ranging from 22 
to 32 days were detected and all these periods are time variable.

Solar activity is generated independently in the two hemispheres and is controlled by differential rotation and meridional circulation in each hemisphere. However, a phase lag of activity in the $\mathrm{N}-\mathrm{S}$ hemisphere typically ranges between a few months to a year or two. This suggests that the coupling between the two hemispheres is variable and weakly coupled (Norton \& Gallagher 2010). We assume that randomness in the Babcock-Leighton process of formation of the poloidal field from the decay of tilted bipolar sunspots introduces asymmetry into the solar cycle. The spatio-temporal asymmetry of meridional flow will also play a key role in the hemispheric asymmetry. More observations of local helioseismology; zonal flow patterns; solar cycle variation of meridional circulation, especially its dynamics below the convection zone; and exact solution of solar dynamo models, whether it is dipolar or quadrupolar, are called for to address the physics of the $\mathrm{N}-\mathrm{S}$ asymmetry precisely. This will improve our knowledge of the origin of asymmetry and the variation of the solar magnetic field.

We thank the anonymous referee for careful reading of the manuscript and constructive comments that improved the original version of the manuscript. We acknowledge the use of Sunspot area data from Marshall space Flight Centre, NASA. Wavelet software provided by C. Torrence and G. Compo are also gratefully acknowledged. Much of this work was done when one of us (P.C.) was an Academic associate at the Inter University Centre for Astronomy and Astrophysics (IUCAA), Pune. P.C. thanks the members of the IUCAA for their warm hospitality. National Solar Observatory (NSO) is operated by the Association of Universities for Research in Astronomy, AURA Inc. under cooperative agreement with the National Science Foundation (NSF).

\section{REFERENCES}

Atac, T., \& Ozguc, A. 1996, SoPh, 166, 201

Badalyan, O. G., Obridko, V. N., \& Sýkora, J. 2008, SoPh, 247, 379

Badalyan, O. G., \& Obridko, V. N. 2011, NewA, 16, 357

Ballester, J. L., Oliver, R., \& Carbonell, M. 2002, ApJ, 566, 505

Ballester, J. L., Oliver, R., \& Carbonell, M. 2005, A\&A, 431, L5

Bai, T. 2003, ApJ, 591, 406

Basu, S., \& Antia, H. M. 2003, ApJ, 585, 553

Basu, S., \& Antia, H. M. 2010, ApJ, 717, 488

Brooke, J. M., Pelt, J., Tavakol, R., \& Tworkowski, A. 1998, A\&A, 332, 353

Carbonell, M., Oliver, R., \& Ballester, J. L. 1993, A\&A, 274, 497

Carbonell, M., Terradas, J., Oliver, R., \& Ballester, J. L. 2007, A\&A, 476, 951

Charbonneau, P. 2005, LRSP, 2, lrsp-2005-2

Chatterjee, P., \& Choudhuri, A. R. 2006, SoPh, 239, 29

Chatterjee, P., Nandy, D., \& Choudhuri, A. R. 2004, A\&A, 427, 1019

Choudhuri, A. R. 1992, A\&A, 253, 277

Choudhuri, A. R., Chatterjee, P., \& Jiang, J. 2007, PhRvL, 98, 131103

Choudhuri, A. R., Schussler, M., \& Dikpati, M. 1995, A\&A, 303, L29

Chowdhury, P., \& Ray, P.C. 2006, MNRAS, 373, 1577

Chowdhury, P., Khan, M., \& Ray, P. C. 2009, MNRAS, 392, 1159

Cowling, T. G. 1934, MNRAS, 94, 768
Donner, R., \& Thiel, M. 2007, A\&A, 475, L33

Dikpati, M., \& Charbonneau, P. 1999, ApJ, 518, 508

Dikpati, M., de Toma, G., \& Gilman, P. A. 2006, GeoRL, 33, L05102

Dikpati, M., de Toma, G., Gilman, P. A., Arge, C. N., \& White, O. R. 2004, ApJ, 601,1136

Dikpati, M., \& Gilman, P. A. 2001, ApJ, 559, 428

Dikpati, M., \& Gilman, P. A. 2006, ApJ, 649, 498

Duchlev, P. I. 2001, SoPh, 199, 211

Durney, B. R. 1995, SoPh, 160, 213

Durrant, C. J., \& Wilson, P. R. 2002, SoPh, 214, 38

Gaizauskas, V., Mackay, D., \& Harvey, K. L. 2001, ApJ, 558, 888

Gao, P. X., Li, K.-J., \& Shi, X.-J. 2009, MNRAS, 400, 1383

Gigolashvili, M. Sh., Japaridze, D. R., Mdzinarishvili, T. G., \& Chargeishvili, B. B. 2005 , SoPh, 227,27

Gnevyshev, M. N. 1963, SvA, 7, 311

Goel, A., \& Choudhuri, A. R. 2009, RA\&A, 9, 115

Guerrero, G. A., \& Muñoz, J. D. 2004, MNRAS, 350, 317

Hathaway, D. H. 2010, LRSP, 7, 1

Hathaway, D. H., \& Rightmire, L. 2010, Sci, 327, 1350

Hathaway, D. H., \& Rightmire, L. 2011, ApJ, 729, 80

Horne, J. H., \& Baliunas, S. L. 1986, ApJ, 302, 757

Howe, R., Christensen-Dalsgaard, J., Hill, F., et al. 2000, Sci, 287, 2456

Janardhan, P., Bisoi, S. K., \& Gosain, S. 2010, SoPh, 267, 267

Jiang, J., Chatterjee, P., \& Choudhuri, A. R. 2007, MNRAS, 381, 1527

Kirk, M. S., Pesnell, W. D., Young, C. A., \& HessWebber, S. A. 2009, SoPh, 257, 99

Knaack, R., Stenflo, J. O., \& Berdyugina, S. V. 2004, A\&A, 418, L17

Knaack, R., Stenflo, J. O., \& Berdyugina, S. V. 2005, A\&A, 438, 1067

Larson, H. J. 1982, Introduction to Probability Theory and Statistical Inference (3rd ed.; New York: John Wiley and Sons, Inc.)

Lean, J. L., \& Brucekner, G. E. 1989, ApJ, 337, 568

Li, K. J., Chen, H. D., Zhan, L. S., et al. 2009, JGR, 114, A04101

Li, K. J., Liu, X. H., Gao, P. X., \& Zhan, L. S. 2010, NewA, 15, 346

Li, K. J., Wang, J. X., Xiong, S. Y., et al. 2002, A\&A, 383, 648

Li, K. J., Yun, H. S., \& Gu, X. M. 2001, ApJL, 554, L115

Lomb, N. R. 1976, Ap\&SS, 39, 447

Lou, Y. Q. 2000, ApJ, 540, 1102

McComas, D. J., Ebert, R. W., Elliott, H. A., et al. 2008, GeoRL, 35, 18103

Mininni, P. D., \& Gomez, D. O. 2002, ApJ, 573, 454

Moss, D. S., Steven, H., \& Sokoloff, D. 2008, MNRAS, 388, 416

Norton, A. A., \& Gallagher, J. C. 2010, SoPh, 261, 193

Nandy, D., \& Choudhuri, A. R. 2002, Sci, 296, 1671

Oliver, R., \& Ballester, J. L. 1994, SoPh, 152, 481

Oliver, R., Ballester, J. L., \& Carbonell, M. 1992, SoPh, 137, 141

Ossendrijver, M. 2003, A\&AR, 11, 287

Pap, J., Tobiska, W. K., \& Bouwer, S. D. 1990, SoPh, 129, 165

Scargle, J. D. 1982, ApJ, 263, 835

Schrijver, C. J., \& Liu, Y. 2008, SoPh, 252, 19

Simoniello, R., Finsterle, W., Salabert, D., et al. 2012, A\&A, 539, A135

Sokoloff, D., \& Nesme-Ribes, E. 1994, A\&A, 288, 293

Sturrock, P. A. 2008, SoPh, 252, 221

Sturrock, P. A., Scargle, J. D., Walther, G., \& Wheatland, M. S. 1999, ApJL, 523, L177

Svalgaard, L., Cliver, E. W., \& Kamide, Y. 2005, GeoRL, 32, L01104

Sýkora, J., \& Rybák, J. 2010, SoPh, 261, 321

Temmer, M., Rybák, J., Bendík, P., et al. 2006, A\&A, 447, 735

Temmer, M., Veronig, A., \& Hanslmeier, A. 2002, A\&A, 390, 707

Torrence, C., \& Compo, G. P. 1998, BAMS, 79, 61

Vizoso, G., \& Ballester, J. L. 1990, A\&A, 229, 540

Wang, Y.-M., Robbrecht, E., \& Sheeley, N. R., Jr. 2009, ApJ, 707, 1372

Wang, Y.-M., Sheeley, N. R., Jr., \& Nash, A. G. 1991, ApJ, 383, 431

Zaatri, A. A., Komm, R., González Hernández, I., Howe, R., \& Corbard, T. 2006, SoPh, 236, 227

Zaqarashvili, T. V., Carbonell, M., Oliver, R., \& Ballester, J.L. 2010a, ApJ, 709,749

Zaqarashvili, T. V., Carbonell, M., Oliver, R., \& Ballester, J. L. 2010b, ApJ, 724, 95 